\title{
TRANS-SCLERAL FIXATION OF DISLOCATED POSTERIOR CHAMBER INTRAOCULAR LENSES USING A 9/0 MICROSURGICAL POLYPROPYLENE SNARE
}

\author{
B. C. LITTLE, P. H. ROSEN, G. ORR and G. W. AYLWARD \\ London
}

\begin{abstract}
SUMMARY
We describe a new technique for retrieval, sulcus relocation and trans-scleral fixation of posteriorly dislocated (posterior chamber intraocular lenses (PC-IOLs) in a closed eye using a 9/0 polypropylene snare instrument adapted specifically for this purpose. Our experience is described of the first 5 consecutive cases in which the snare was used. The posteriorly dislocated PC-IOL was successfully relocated in 4 of these cases but per-operative fracture of a lens haptic necessitated removal of the IOL in the fifth. A number of surgical techniques have already been described in the management of this problem but we consider the polypropylene snare to offer the advantages of a simpler technique that obviates the need for either manipulation of needles within the eye or complex suturing procedures.
\end{abstract}

Posterior chamber intraocular lens subluxation in all its forms, including decentration, occurs in about $1 \%$ of cases. ${ }^{1}$ Total posterior dislocation of the PC-IOL into the vitreous cavity is a much rarer event. It presents the surgeon with a complex problem to which, in principle, there are three solutions: to leave the IOL in the vitreous and correct the refractive error with either a contact lens or a secondary anterior chamber IOL (AC-IOL); to remove the PC-IOL and either exchange it for an AC-IOL or correct the aphakia with a contact lens or a secondary IOL; to reposition the dislocated PC-IOL and trans-sclerally fixate the haptics to the globe. Each case needs to be assessed on an individual basis. Indications for surgery include the presence of persistent inflammation, cystoid macular oedema, vitreous incarceration in the cataract wound or retinal detachment. ${ }^{2}$ We consider that a closed-eye technique of IOL repositioning and trans-scleral fixation is for most individuals the safest alternative that offers the best chance of visual rehabilitation.

Various ingenious and innovative techniques have recently been described for repositioning a dislocated PCIOL..$^{3-9}$ The principal advantage of our technique, which

Correspondence to: B. C. Little, Moorfields Eye Hospital, City Road, London EC1V 2PD, UK. we consider to be a significant one, is that the IOL can be secured and manipulated in the eye with the snare instrument, which uses the same suture to grip the IOL and to secure the lens haptic to the sclera. The only instrument to enter the eye during lens relocation and suturing, other than the snare, is a pair of intraocular forceps.

\section{METHOD}

The snare consists of three components: a titanium handle containing a sliding piston under fingertip control via a set-screw, a 23 gauge stainless steel Luer-lock cannula and a $9 / 0$ polypropylene suture snare crimped into a fine steel tube (Fig. 1). The cannula locates on the tapered tip of the handle and the snare is fed through it into the piston and locked in place by tightening the screw. The snare can now be opened and closed by sliding the fingertip control in either direction. With the snare in the open position it is looped around the lens haptic (Fig. 2). It is then closed, thereby tightening the snare loop around the haptic and securing the IOL (Fig. 3).

The eye is prepared for a three-port pars plana vitrectomy (PPV) using a $4 \mathrm{~mm}$ long infusion cannula in the inferotemporal quadrant and two entry ports are made at 9.30 and 2.30 o'clock. Two partial-thickness scleral flaps which overlie the ciliary sulcus are fashioned diametrically opposite each other and a 9/0 polypropylene loop is pre-placed in the base of each flap using a quarter circle needle which is cut off to leave two free ends of polypropylene under each flap (Fig. 4). These act as separate pre-placed sutures to which the ends of the snares that will hold each haptic in place are later tied. A vitrectomy is performed so that the IOL is freely mobile and exerting no vitreoretinal traction.

An entry port to accommodate the snare cannula is made under each flap at the ciliary sulcus using a 23 gauge needle. The exact distance of the entry site from the posterior border of the surgical limbus varies according to the clock-hour position of the scleral flap. Duffy ${ }^{8}$ has shown in cadaveric eyes that the sulcus is located on average $0.83 \pm 0.1 \mathrm{~mm}$ from the posterior surgical limbus in the 


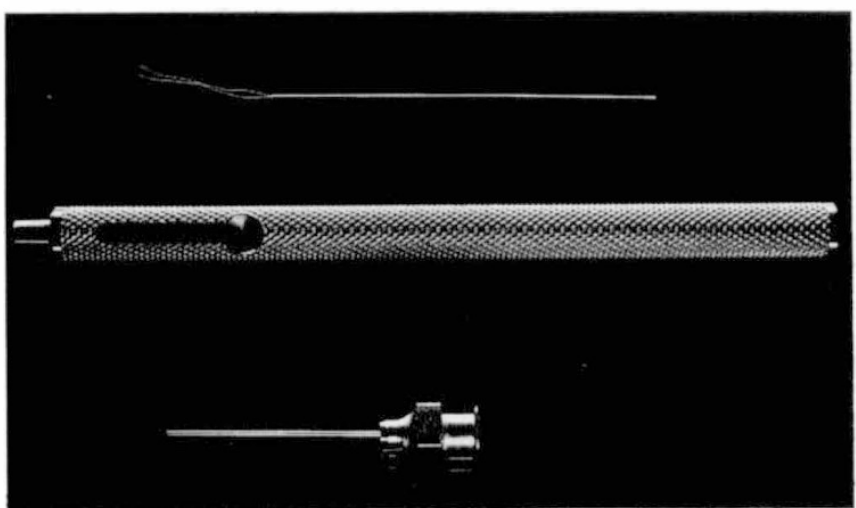

Fig. 1. The three components of the microsurgical polypropylene snare instrument.

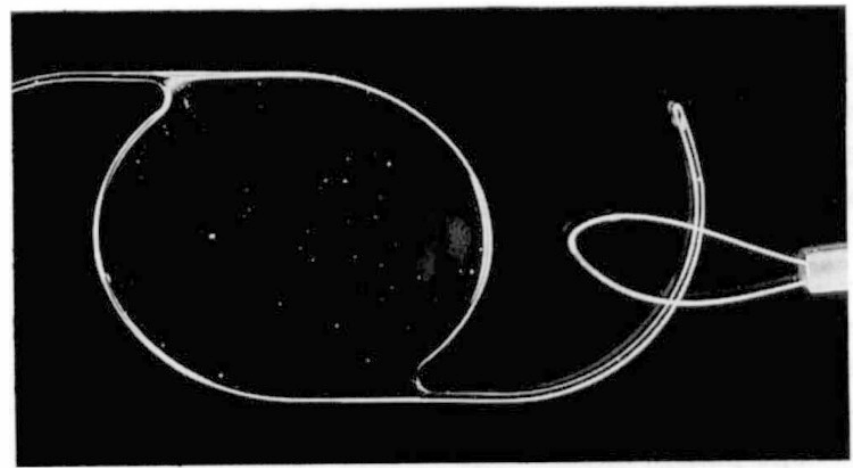

Fig. 2. The snare in the open position passed around an IOL haptic.

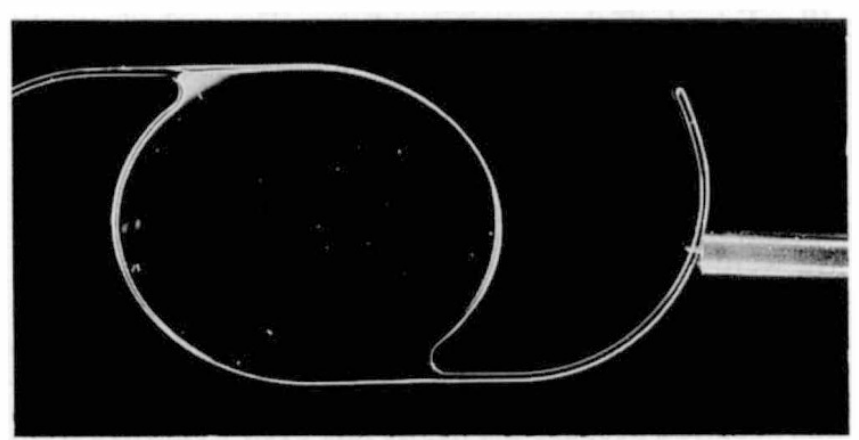

Fig. 3. The snare in the closed position firmly grasping the IOL haptic up against the end of the cannula.

vertical meridian and $0.46 \pm 0.1 \mathrm{~mm}$ in the horizontal meridian.

The snare, in its closed position, is inserted through one of the entry sites. If the IOL is lying flat against the retina and the snare cannot readily be passed around the end of the haptic, then the least traumatic method of lifting it off the retina is with the use of perfluorocarbon 'heavy liquid'. The snare can then be looped around the first haptic. Using the fingertip slide, the snare is closed, drawing the haptic firmly up against the end of the cannula thereby securing the IOL and enabling positive control of its position. By withdrawing the cannula from the eye the haptic is guided into the ciliary sulcus. Once the haptic is located then the retaining screw on the fingertip slide is loosened, thereby allowing the cannula to be withdrawn over the snare which is grasped with plain forceps at its site of entry into the

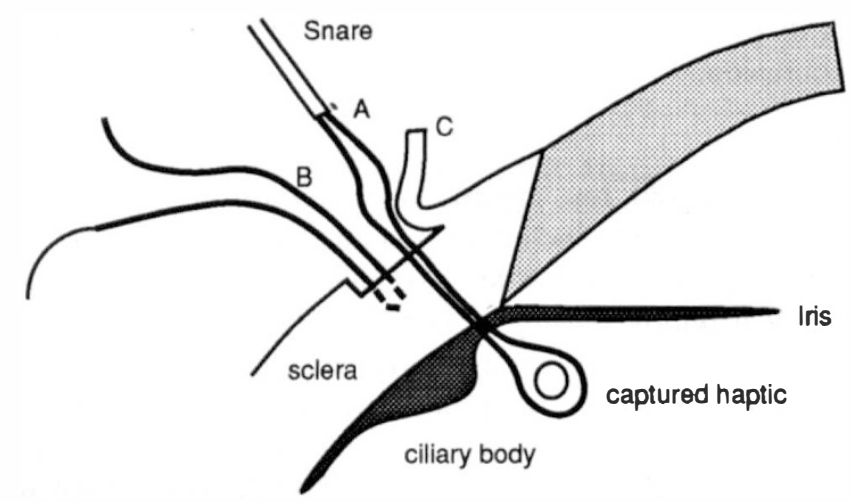

Fig. 4. Diagram showing the relationship and location of the snare $(A)$ and pre-placed suture $(B)$ under the scleral flap $(C)$.

sclera. As the cannula is further withdrawn over the snare, a bulldog clip is placed near the root of the snare and it is cut from the retaining tube at its origin. The weight of the bulldog clip applies continuous traction to the ends of the polypropylene and so reduces the chance of the ensnared haptic slipping out of the loop.

The second haptic is relocated in an identical manner to the first. After removing the bulldog clip from the first snare/suture, it is tied to the pre-placed polypropylene loop to secure the haptic in place, i.e. two ends tied to two ends in a single knot. The process is repeated for the second haptic and the scleral flaps are closed with 10/0 nylon to cover the knots.

\section{RESULTS}

\section{Case 1}

An 80-year-old man underwent right intercapsular cataract extraction complicated by posterior capsular dehiscence and vitreous loss. An anterior vitrectomy was performed and the surgeon felt that there was sufficient anterior capsular support to place a PC-IOL in the ciliary sulcus. Whilst adjusting the position of the IOL it sank back into the vitreous cavity and attempts at primary retrieval were unsuccessful so the eye was closed.

Assessment 2 days later by the vitreoretinal service revealed retinal contusion, from impact of the IOL, reducing the corrected acuity to $6 / 24$. The eye was allowed to settle for 2 weeks before undergoing three-port PPV, IOL retrieval, sulcus relocation and trans-scleral fixation.

The first haptic was successfully relocated and sutured using the technique as described above. The 23 gauge paracentesis for snaring the second haptic was probably not fully penetrative since attempted introduction of the snare was unsuccessful and there was a suspicion of a small cyclodialysis that may have been caused by repeated attempts at entry. The paracentesis was enlarged but it was thought to be unwise to attempt further introduction of a blunt instrument so a trans-corneal 9/0 prolene loop was threaded across the anterior chamber into a 27 gauge needle which had been passed through the entry site.

The IOL was secured and well centred at the end of surgery. Although the IOL remained well positioned within the 2 month follow-up period, the vision remained down 
at its pre-operative level of $6 / 24$ due to resolving retinal contusion.

\section{Case 2}

A 68-year-old low myope had previously undergone multiple surgery including silicone oil exchange for a giant retinal tear in her left eye. She developed a cataract that was removed using an intracapsular technique with a silicone oil top-up. The retina re-detached 1 month later but was flat following vitrectomy, endolaser, membrane peel and further oil top-up. Five months later the retina remained re-attached and the patient underwent removal of silicone oil combined with a secondary sulcus-sutured PC-IOL.

Eguchi sutures (9/0 polypropylene loops on a curved needle) were tied at the apex of the IOL haptics and the needles of each passed via the corneal section into the anterior chamber and through the pupil to be fed into a 27 gauge needle which had entered the eye externally at the ciliary sulcus. The suture was then withdrawn from the eye and the first haptic secured to a scleral pre-placed 9/0 prolene suture. As the suture holding the second haptic was being placed under tension prior to tying, it broke and the IOL swung backwards into the vitreous cavity hinged on the first haptic. The $9 / 0$ polypropylene snare was introduced through the sulcus entry site and looped around the free haptic; then the IOL was retrieved, relocated in the sulcus and trans-sclerally fixated without opening the eye.

Six months post-operatively the patient's corrected vision in this eye was $6 / 12$ with a well-centred IOL.

\section{Case 3}

A 44-year-old insulin-dependent diabetic with a renal transplant and systemic hypertension had bilateral proliferative diabetic retinopathy that had been previously lasered, but he re-presented with recurrent vitreous haemorrhage in both eyes. He had a cataract in his left eye in which he had also developed open angle glaucoma that was poorly controlled on medical treatment. Pre-operative acuity in his left eye was hand movements. He underwent left PPV combined with trabeculectomy, extracapsular cataract extraction and IOL implantation. No per-operative complications were noted.

On day 1 no IOL was seen and the eye was soft. On day 2 the IOL was located in the vitreous and there were extensive choroidal effusions. Further surgery was therefore postponed. One week later the patient suffered a spontaneous suprachoroidal haemorrhage which settled over the following month. He then underwent further vitrectomy combined with membrane peel and IOL repositioning using the snare technique for sulcus fixation of both haptics.

At 1 month follow-up the IOL was well centred and stable but the trabeculectomy was failing and the patient's visual acuity poor (2/60) due to his diabetic retinopathy.

\section{Case 4}

A 64-year-old man had previously been punched in his left eye during an assault; this resulted in a hyphaema and over the subsequent 5 years a cataract developed.

The patient underwent uneventful endocapsular cataract surgery. On day 1 his visual acuity was 6/9 but the IOL was decentred temporally. Two weeks later he noticed monocular diplopia and the lens was further decentred temporally into the anterior vitreous, which had now prolapsed in front of the IOL.

PPV and IOL repositioning were performed using the snare technique for sulcus fixation of the first haptic. There was sufficient residual capsular remnant on the nasal side to stabilise the second haptic in the ciliary sulcus without the need for trans-scleral fixation.

At 3 months follow-up the lens was well centred and acuity matched the pre-operative level of 6/9.

\section{Case 5}

A 76-year-old bilaterally pseudophakic man had previously undergone phacoemulsification complicated by posterior capsular rupture in both eyes in which sulcuslocated IOLs were implanted. Corrected visual acuities of 6/9 left and 6/12 right were achieved.

Seven months after surgery to his second eye he tripped and fell, sustaining a blow to the left side of his head. He noticed a rapid loss of vision in his left eye and on examination the IOL was dislocated into the vitreous cavity.

The patient underwent PPV and IOL relocation using the snare. The first haptic was snared and relocated without difficulty, but the second haptic fractured at its apex during relocation (Fig. 5) and the 9/0 loop suture repeatedly slipped off the remaining stump. The procedure was therefore abandoned and the IOL removed through a corneal section. The eye was left aphakic with a plan to use contact lens correction at a later date.

\section{DISCUSSION}

We were able successfully to retrieve, relocate and transsclerally fixate 4 of our first 5 cases of posteriorly dislocated PC-IOLs using this new technique. The fifth had to be abandoned after one of the haptics fractured: whether this occurred because the haptic had been weakened from stresses inflicted during previous implant surgery or was caused de novo during relocation remains unclear.

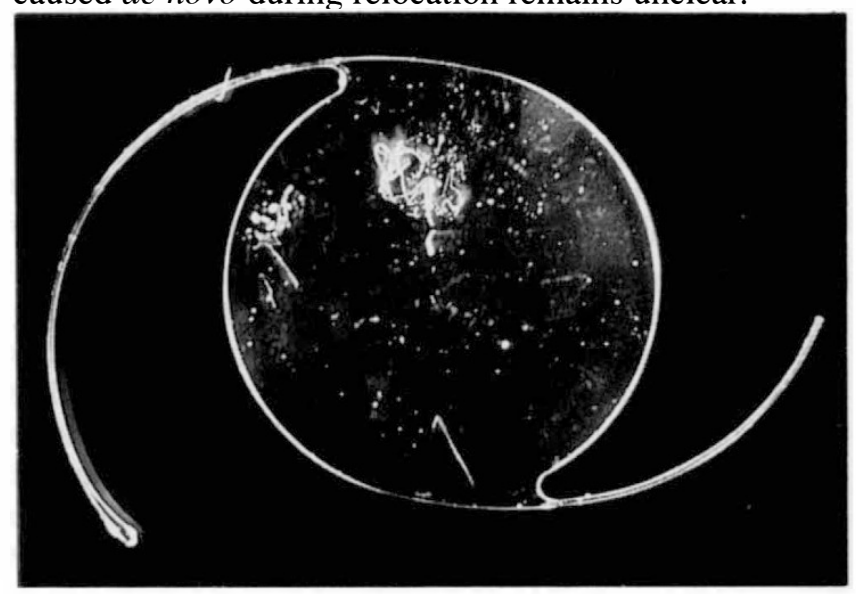

Fig. 5. IOL removed following fracture of the haptic (case 5). 
During the first case, considerable difficulty was experienced attempting to introduce the snare cannula through the 23 gauge entry site, which required enlarging with an MVR blade. At this point it was felt wisest not to reattempt the introduction of a blunt cannula through this port because of the possibility of the presence of a small choroidal detachment or cyclodialysis cleft. Review of the operation video showed that the 23 gauge needle had not penetrated the eye sufficiently deeply to create an adequate entry site.

It is important either to bury the polypropylene suture in the scleral wound, or to create scleral flaps for this purpose as we do in our technique, together with meticulous trimming of the suture ends, since endopthalmitis secondary to suture erosion through the conjunctiva has been reported. ${ }^{10}$

One scleral suture per haptic has been found experimentally to be adequate for stable fixation and centration of the $\mathrm{IOL}^{8}$ obviating the need for double-armed suture passes recommended by others ${ }^{11-13}$ and lessening the risk of haemorrhage.

It is our impression that the incidence of IOLs dislocated into the vitreous cavity may be increasing. Precisely why this should be is unclear, but perhaps may be related to an increasing use of continuous tear capsulorrhexis. In the presence of a capsulorrhexis there is probably greater confidence about implanting posterior chamber lenses into the sulcus when a posterior capsular tear has occurred. However, unless the peripheral limit of the capsular tear is visible it is impossible to know the extent of the tear and the subsequent stability of any IOL located in the ciliary sulcus.

We believe that the snare technique offers a simple and versatile method of retrieving, relocating and trans-sclerally suturing a posteriorly dislocated IOL using minimal intraocular instrumentation and manipulation.
Key words: Dislocated intraocular lens, Microsurgical snare, Transscleral fixation.

\section{REFERENCES}

1. Stark WJ, Bruner WE, Martin NF. Management of dislocated subluxed posterior chamber intraocular lenses. Ophthalmic Surg 1982;13:130-3.

2. Brod RD, Flynn HW Jr, Clarkson JG, et al. Management options for retinal detachment in the presence of a posteriorly dislocated intraocular lens. Retina 1990;10:50-6.

3. Sternberg P, Michels RG. Treatment of dislocated posterior chamber intraocular lenses. Arch Ophthalmol 1986;104: 1391-3.

4. Insler MS, Mani H, Peyman GA. A new surgical technique for dislocated posterior chamber intraocular lenses. Ophthalmic Surg 1988;19:480-1.

5. Smiddy WE. Dislocated posterior chamber intraocular lens: a new technique of management. Arch Ophthalmol 1989; 107:1678-80.

6. Bloom SM, Wyszynski RE, Brucker AJ. Scleral fixation suture for dislocated posterior chamber intraocular lens. Ophthalmic Surg 1990;21:851-4.

7. Campo RV, Chang KD, Oyakawa RT. Pars plana vitrectomy in the management of dislocated posterior chamber intraocular lenses. Am J Ophthalmol 1989;108:529-34.

8. Duffy RJ, Holland EJ, Agapitos PJ, Lindstrom RL. Anatomic study of trans-sclerally sutured intraocular lens implantation. Am J Ophthalmol 1989;108:300-9.

9. Smiddy WE, Flynn HW Jr. Management of retained lens nuclear fragments and dislocated posterior chamber intraocular lenses after cataract surgery. Semin Ophthalmol 1993;8:96-103.

10. Heilskov T, Joondeph BC, Olsen KR, Blankenship GW. Late endophthalmitis after trans-scleral fixation of a posterior chamber intraocular lens. Arch Ophthalmol 1989;107: 1427.

11. Stark WJ, Goodman G, Goodman B, Gottsch J. Posterior chamber intraocular lens implantation in the absence of posterior capsule support. Ophthalmic Surg 1988;19:240.

12. Hu BV, Shin DH, Gibbs KA, YJ. Implantation of posterior chamber lens in the absence of zonular and capsular support. Arch Ophthalmol 1988;106:416.

13. Girard L. PC-IOL implantation in the absence of posterior capsular support. Ophthalmic Surg 1988;19:680. 